\title{
Putting insulin resistance into context by dietary reversal of type 2 diabetes
} R Taylor ${ }^{1}$

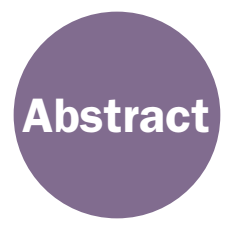

It has long been assumed that type 2 diabetes is caused by the combined effects of insulin resistance and loss of pancreatic islet beta cells. Much therapeutic effort has been directed towards decreasing insulin resistance even though results have been generally disappointing. Now that type 2 diabetes can be understood as a simple reversible condition, its component parts can be examined during the period of return to normal glucose tolerance.

\section{Correspondence to: R Taylor Magnetic Resonance Centre Campus for Ageing \& Vitality Newcastle University Newcastle upon Tyne NE4 5PL UK}

\section{Email:}

roy.taylor@ncl.ac.uk

Based on a lecture given at the Diabetes and Endocrinology symposium, Royal College of Physicians of Edinburgh, October 2016

\section{What is insulin resistance?}

Any individual will respond to a fixed dose of insulin in a manner depending upon the degree of sensitivity to insulin. At the one end of the spectrum, slim athletic people are likely to be very sensitive to insulin, whereas obese inactive people are likely to be relatively insensitive, or insulin resistant. Different tissue exhibit different sensitivity to insulin, but the phrase 'insulin resistance' has become synonymous with results of euglycemic hyperinsulinemic clamp studies. These tests predominantly measure muscle insulin sensitivity. ${ }^{1}$

Insulin resistance in muscle is the earliest indicator of risk of future development of type 2 diabetes. ${ }^{2}$ It has been assumed that reversing the insulin resistance would considerably improve the level of blood glucose control in established type 2 diabetes, and much therapeutic effort has been directed at the targeted insulin resistance. However, even vigorous exercise training produces only a modest effect on blood glucose control. ${ }^{3}$ Similarly, drugs developed specifically to improve insulin resistance - thiazolidinediones - produce only modest benefit. ${ }^{4}$

It is the insulin sensitivity of a different organ which is most closely associated with control of fasting plasma glucose. The liver is responsible for continuous production of glucose. This is essential to survival as the brain requires a continuous supply of glucose for its energy needs in everyday life. Especially during the overnight period, life continues only because the liver produces around $10 \mathrm{~g}$ of glucose per hour. ${ }^{5}$ This is regulated by insulin, and hence any degree of insulin resistance in this process would lead to increased production of glucose. Indeed, fasting plasma insulin is typically raised in type 2 diabetes, but nonetheless the liver produces around $15 \mathrm{~g}$ of glucose per hour. ${ }^{6}$

\section{Predictions of the twin cycle hypothesis}

In 2008 it was possible to bring together information on control of liver glucose production and beta cell function to postulate the events leading to the onset of type 2 diabetes (Figure 1). ${ }^{7}$

Type 2 diabetes only occurs after a decade or more of positive energy balance. Any fat in excess of daily requirement for oxidation can be stored in subcutaneous tissue but any excess carbohydrate can be handled only by one pathway: conversion to triglyceride. This happens solely in the liver and is promoted by the presence of high insulin levels. The pathway is not subject to insulin resistance, so individuals who are relatively insulin resistant have modestly raised plasma insulin levels and will be able to convert carbohydrate into liver fat more readily. The first step in the twin cycle hypothesis is that the levels of fat inside the liver gradually rise and this brings about increasing liver insulin resistance. In order to control the output of glucose by the liver, plasma insulin levels must rise. It creates a vicious cycle leading to steadily increasing liver fat levels.

The higher the liver fat level, the higher the rate of export of triglyceride to the rest of the body. This is done in the form of 
During chronic positive calorie balance, any excess carbohydrate must undergo de novo lipogenesis. There is no other available pathway, and it can only occur in the liver. This particularly promotes fat accumulation in the liver. As insulin stimulates de novo lipogenesis, individuals with a degree of insulin resistance (determined by family or lifestyle factors) will increase liver fat more readily than others due to the higher plasma insulin levels. The increased liver fat will cause relative resistance to suppression of hepatic glucose production by insulin. Over many years, this will bring about a small increase in fasting plasma glucose level, and hence increased basal insulin secretion rates. The consequent hyperinsulinemia will further enhance the conversion of excess calories into liver fat. A vicious cycle of hyperinsulinemia and blunted suppression of hepatic glucose production becomes established. Fatty liver leads to increased export of VLDL triacylglycerol ${ }^{21}$ which will increase fat delivery to the islets of the pancreas along with all other tissues. This process is further stimulated by elevated plasma glucose levels. ${ }^{21}$ Excess fatty acid availability in the pancreatic islet will bring about beta-cell de-differentiation ${ }^{22-24}$ and impair the acute insulin secretion in response to ingested food. At a certain level of fatty acid exposure, postprandial hyperglycaemia will develop. The hyperglycaemia will further increase insulin secretion rates and hepatic lipogenesis, spinning the liver cycle faster and promoting the pancreas cycle. Eventually the fatty acid and glucose inhibitory effects on the islets reach a trigger level leading to a relatively sudden onset of clinical diabetes..$^{25}$ Figure adapted with permission from Taylor ${ }^{7}$ and reproduced with permission from Taylor. ${ }^{26}$

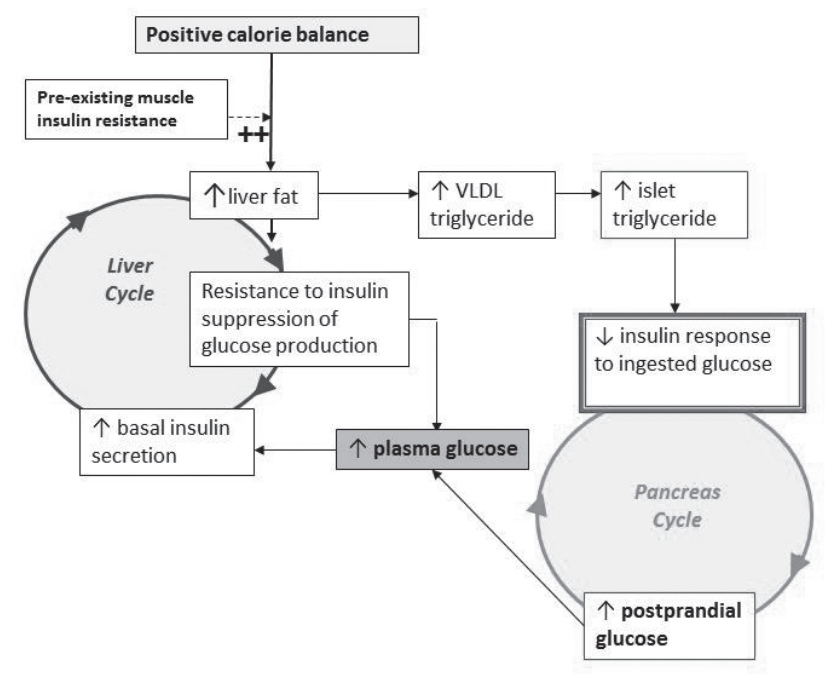

\section{But what happened to muscle insulin resistance during the disappearance of the type 2 diabetic state?}

take up excess triglyceride supply avidly is the islet tissue of the pancreas. ${ }^{8-10}$ Early data indicated that increasing levels of fat in the pancreas could decrease the acute insulin response to a raised glucose level. ${ }^{9}$ The diminishing real time insulin response would have the effect of producing greater postprandial hyperglycaemia, with prolongation of high insulin levels after meals, and steady onward progress of both the vicious cycles affecting liver and pancreas.

\section{Observations during reversal of type 2 diabetes}

The twin cycle hypothesis permitted testing of each of the predictions. The Counterpoint study followed a group of people with very ordinary type 2 diabetes during negative calorie balance. According to the hypothesis, the need to oxidise endogenous stored fuel should make the twin vicious cycles spin in the opposite direction. Oral hypoglycaemic agents were stopped on day 1 of the low calorie diet. Within 7 days liver fat levels had fallen by $30 \%$, liver insulin sensitivity had normalised, and fasting plasma glucose had normalised. Then, over the subsequent 8 weeks, the level of fat within the pancreas gradually decreased, and first phase insulin response gradually returned. ${ }^{11}$

The later observation was striking and definitive. For decades, it had been assumed that first phase insulin response could never be restored in type 2 diabetes. Overall, the events predicted by the twin cycle hypothesis were shown to occur during an 8 week period of low calorie diet and weight loss of $15 \mathrm{~kg}$. Both cycles could be made to spin in reverse.
Euglycemic hyperinsulinemic clamp studies during the Counterpoint study allowed measurement of muscle insulin resistance, separate from that of the liver. No change in muscle insulin resistance occurred despite return to the non-diabetic state. ${ }^{11}$ Clearly, reversal of the muscle insulin resistance was not required to achieve normal glycaemia.

\section{Longer term observations}

It was necessary to conduct a further study to observe the long term physiology after reversal of type 2 diabetes. The Counterbalance study was designed to follow up individuals for six months after initial weight loss. ${ }^{12,13}$ It confirmed the findings of the Counterpoint study. In particular it showed that there was no change in muscle insulin resistance after reversal of type 2 diabetes, and only a trivial improvement over the six months of weight maintenance and continuation of non-diabetic glucose control.

\section{The essential nature of type 2 diabetes}

It can now be seen that muscle insulin resistance is a factor which expedites the accumulation of liver fat during the phase of developing the condition. Muscle insulin resistance itself is not relevant to maintenance of the diabetic state (see Box 1). The cellular pathology of beta cell dysfunction in type 2 diabetes is now amenable to a simple explanation. Presence of excess fat causes endoplasmic reticulum stress. This causes the beta cell to go into a 'survival' mode during which non-essential functions are shutdown. Producing insulin is not 
Box 1 Muscle insulin resistance

- Mice totally lacking muscle receptors do not develop diabetes ${ }^{18}$

- People with PP1R2A variant of glycogen synthase cannot store glucose as glycogen and muscle - yet have normal glucose tolerance ${ }^{19}$

- $8 \%$ weight loss in type 2 diabetes markedly improved glucose control but with no change in muscle insulin resistance ${ }^{20}$

- $15 \%$ weight loss normalises glucose homeostasis in type 2 diabetes with no decrease in muscle insulin resistance ${ }^{11,12}$

essential for the survival of the beta cell and, unsurprisingly, the insulin gene production is switched off in this survival mode. However, at least for a period of a few years, these changes are reversible and removal of the excess fat allows redifferentiation of the beta cell. This sequence of events can now be described in relation to observations of physiology of reversal of type 2 diabetes. ${ }^{14}$

\section{Insulin resistance in context}

Although insulin resistance is associated with physical inactivity, dyslipidaemia and increased risk of macrovascular disease, it is important to set in context its role in the development of type 2 diabetes.
Given that the degree of insulin resistance exhibited by people with type 2 diabetes overlaps entirely with that of the non-diabetic majority population, ${ }^{15}$ muscle insulin resistance can be seen not as a disease state per se, but as an individual characteristic which affects risk. It does not dictate pathogenesis.

The good news for people with type 2 diabetes is that they have a potentially reversible metabolic condition. Large numbers of individuals who are strongly motivated have now returned their metabolic state to normal, and are off all medication. ${ }^{16}$ While these insights allow motivated people to make a profound difference to their own health, acquiring evidence upon which a change of guideline advice can be based is very different. It remains to be demonstrated how this can be applied to the management of all people with type 2 diabetes. A large study in primary care will test the practical applicability of the approach by randomisation to either weight loss plus a behavioural weight maintenance programme or to best care according to current guidelines. ${ }^{17}$ DiRECT (Diabetes Remission Clinical Trial) will report the one year outcomes this year. (1)

\section{References}

1 DeFronzo RA, Jacot E, Jequier E et al. The effect of insulin on the disposal of intravenous glucose. Results from indirect calorimetry and hepatic and femoral venous catheterisation. Diabetes 1981; 30: 1001-7.

2 Petersen KF, Dufour S, Savage DB et al. The role of skeletal muscle insulin resistance in the pathogenesis of the metabolic syndrome. Proc Natl Acad Sci U S A 2007; 104: 12587-94.

3 Cassidy S, Thoma C, Hallsworth $\mathrm{K}$ et al. High intensity intermittent exercise improves cardiac structure and function and reduces liver fat in patients with type 2 diabetes: a randomised controlled trial. Diabetologia 2016; 59: 56-66.

4 Kim H, Haluzik M, Gavrilova O, Yakar S et al. Thiazolidinediones improve insulin sensitivity in adipose tissue and reduce the hyperlipidaemia without affecting the hyperglycaemia in a transgenic model of type 2 diabetes. Diabetologia 2004; 47: 2215-25.

5 Taylor R, Magnusson I, Rothman DL et al. Direct assessment of liver glycogen storage by $13 \mathrm{C}$ nuclear magnetic resonance spectroscopy and regulation of glucose homeostasis after a mixed meal in normal subjects. J Clin Invest 1996; 97: 126-32.

6 Singhal P, Caumo A, Carey PE et al. Regulation of endogenous glucose production after a mixed meal in type 2 diabetes. Am J Physiol Endocrinol Metab 2002; 283: E275-83.

7 Taylor R. Pathogenesis of type 2 diabetes: Tracing the reverse route from cure to cause. Diabetologia 2008; 51: 1781-9.

8 Lalloyer F, Vandewalle B, Percevault F et al. Peroxisome proliferatoractivated receptor alpha improves pancreatic adaptation to insulin resistance in obese mice and reduces lipotoxicity in human islets. Diabetes 2006; 55: 1605-13.
9 Lee Y, Hirose H, Ohneda M, Johnson JH et al. Beta-cell lipotoxicity in the pathogenesis of non-insulin-dependent diabetes mellitus of obese rats: impairment in adipocyte-B-Cell relationships. Proc Natl Acad Sci U S A 1994; 91: 10878-82.

10 Lee Y, Lingvay I, Szczepaniak LS et al. Pancreatic steatosis: harbinger of type 2 diabetes in obese rodents. Int J Obes 2009; 34: 396-400.

11 Lim EL, Hollingsworth KG, Aribisala BS et al. Reversal of type 2 diabetes: normalisation of beta cell function in association with decreased pancreas and liver triacylglycerol. Diabetologia 2011; 54: $2506-14$.

12 Steven S, Hollingsworth KG, Al-Mrabeh A et al. Very low calorie diet and 6 months of weight stability in type 2 diabetes: pathophysiological changes in responders and nonresponders. Diabetes Care 2016; 39: 808-15.

13 Steven S, Taylor R. Restoring normoglycaemia by use of a very low calorie diet in long versus short duration type 2 diabetes. Diabet Med 2015; 32: 1149-55.

14 White MG, Shaw JAM, Taylor R. Type 2 diabetes: The pathologic basis of reversible beta-cell dysfunction. Diabetes Care 2016; 39: 2080-8.

15 Taylor R. Insulin resistance and type 2 diabetes. Diabetes 2012; 61: 778-9.

16 Steven S, Lim E, Taylor R. Population response to information on reversibility of type 2 diabetes. Diabet Med 2013; 30: e135-8.

17 Leslie WS, Ford I, Sattar N et al. The Diabetes Remission Clinical Trial (DiRECT): protocol for a cluster randomised trial. BMC Fam Pract 2016; 17: 20. 
18 Brüning JC, Michael MD, Winnay JN et al. A muscle-specific insulin receptor knockout exhibits features of the metabolic syndrome of NIDDM without altering glucose tolerance. Mol Cell 1998; 2: 559 69.

19 Savage DB, Zhai L, Ravikumar B et al. A prevalent variant in PPP1R3A impairs glycogen synthesis and reduces muscle glycogen content in humans and mice. PLoS Med 2008; 5: e27.

20 Petersen KF, Dufour S, Befroy D et al. Reversal of nonalcoholic hepatic steatosis, hepatic insulin resistance, and hyperglycemia by moderate weight reduction in patients with type 2 diabetes. Diabetes 2005; 54: 603-8.

21 Adiels M, Taskinen MR, Packard C et al. Overproduction of large VLDL particles is driven by increased liver fat content in man. Diabetologia 2006; 49: 755-65.

22 Pinnick K, Neville M, Clark A et al. Reversibility of metabolic and morphological changes associated with chronic exposure of pancreatic islet beta-cells to fatty acids. J Cell Biochem 2010; 109 : 683-92.
23 Talchai C, Xuan S, Lin HV et al. Pancreatic beta cell dedifferentiation as a mechanism of diabetic beta cell failure. Cell 2012; 150 : 1223-34.

24 White MG, Marshall HL, Rigby R et al. Expression of mesenchymal and alpha-cell phenotypic markers in islet beta-cells in recently diagnosed diabetes. Diabetes Care 2013; 36: 3818-20.

25 Tabak AG, Jokela M, Akbaraly TN et al. Trajectories of glycaemia, insulin sensitivity, and insulin secretion before diagnosis of type 2 diabetes: an analysis from the Whitehall II study. Lancet 2009; 373: 2215-21.

26 Taylor R. Type 2 diabetes: etiology and reversibility. Diabetes Care 2013; 36: 1047-55. 